\title{
Development and implementation of early intervention services for young people with psychosis: case study
}

Helen Lester, Max Birchwood, Stirling Bryan, Elizabeth England, Helen Rogers and Namita Sirvastava

\section{Background}

The development of early intervention services for young people with first-episode psychosis is a priority internationally.

\section{Aims}

To evaluate the development, implementation and impact of existing and newly formed early intervention services in England.

\section{Method}

Multiple-case study involving staff, users, carers and commissioners of 14 early intervention services.

\section{Results}

Service numbers increased in response to national policy directives. They were still actively working with $90.6 \%$ of service users 12 months after inception. They were highly valued by users and carers as providing a personal service that contrasted with previous experiences of care. Tensions between providing a quality service and meeting case-load targets linked to future funding led teams to adopt a series of survival strategies with some unintended consequences.

\section{Conclusions}

Early intervention services are highly valued by consumers and engage users effectively after 12 months. Implementation of these services is threatened unless sufficient consistent funding is made available.

\section{Declaration of interest}

None.
Psychosis can be a devastating illness. It affects $3.4 \%$ of the population over a lifetime. ${ }^{1}$ Eighty per cent of first episodes of psychosis occur between the ages of 16 and 30 years. During the past decade, the development of early intervention services for young people with first-episode psychosis has become a priority in a number of countries, including England, Canada, Australia and Scandinavia. This has occurred in response to a shift in resources and locus of care from hospital- to community-based services, user and carer concerns about existing services ${ }^{2}$ and evidence that community mental health teams are unable to engage young people effectively or provide specific treatments needed during the early critical period of the illness. ${ }^{3}$ A duration of untreated psychosis of 1-2 years ${ }^{4}$ and the benefits to patients of reducing this by intervening earlier also support the need for a distinct approach. ${ }^{5,6}$

Early intervention services aim to provide comprehensive, community-based care for young people experiencing early psychosis. In the UK, the Department of Health's Plan for the National Health Service (NHS) ${ }^{7}$ stated that 50 early intervention services for young people with first-episode psychosis aged 1435 years would be established across England by 2004. Our study aimed to evaluate the development of existing and newly formed early intervention services across the West Midlands of England. It reports the new approaches to care that emphasised access and engagement, the importance of context, the influence of commissioners, strategies for survival in uncertain times and service user and carer views of services.

\section{Method}

\section{Participants}

A multiple-case study approach was used since this is an effective mechanism both for describing the nature and content of a service initiative (including the context in which it has been developed) and for identifying those factors that may explain its success or failure over a period of time. Moreover, case studies are an excellent methodology for examining interlinked complex social relations as they generate rich descriptive and explanatory accounts of organisational processes, work practices, and the impact of change from the perspective of those being studied, which can provide a source of explanation for wider developments. ${ }^{9,10}$ The formative and longitudinal approach also meant we were able to observe the evolution of services, including unanticipated phenomena, and identify and clarify critical issues in service development and implementation.

Semi-structured interviews, each lasting about $1 \mathrm{~h}$, were undertaken with each early intervention service team manager and most team members at three time points: Spring 2004, Autumn 2004 and Spring 2005. In Autumn 2005, interviews were carried out with team managers alone to clarify emerging themes. Mental health leads and commissioners for each of the four strategic health authorities, 17 of 30 primary care trusts and 5 mental health and social care trusts within the region were interviewed at one time point in 2004-5. Early intervention service users and carers were interviewed at one time point in 2005-6. Written documentation such as annual reports and standard operating procedures were also collected. Each service was also asked to complete a 64 -item scale with items drawn from national policy implementation guidance ${ }^{11}$ that focused on team structure and treatment availability, to explore intervention fidelity.

Standard demographic data were collected on each of the incepted services users during the study period to help describe workload. In view of the importance attached by team members to service engagement, during the first round of interviews we also collected data on the extent of service engagement as recorded in care records at 12 months. 


\section{Procedure and topic guide}

All topic guides were developed from the literature and a priori ideas and piloted prior to use. Topic guides for user and carers were additionally developed in consultation with members of established mental health user and carer groups.

Service users were contacted through keyworkers to ensure that they were well enough to be approached for interview. Within this sampling frame, purposeful sampling was used to interview service users with a range of demographic characteristics. Participating service users were asked to nominate a carer to be interviewed. No approach was made without their consent. Consent from all participants to publish comments was obtained. Ethical approval was granted by the South West Multi Centre Research Ethics Committee

\section{Analysis}

Interviews were tape-recorded and fully transcribed. Data collection and analysis were concurrent and continued until data saturation was complete. Two members of the research team read each transcript. A constant comparison method was used to help interpret the data. ${ }^{12} \mathrm{~A}$ decision was made early on in the data collection process not to use qualitative software since all authors were responsible for collecting and analysing the entire data-set and so felt sufficiently immersed in the data to code and interpret appropriately. Key concepts and categories were identified using an open coding method by deconstructing each interview sentence by sentence. Main categories were then compared across interviews and reintegrated into common themes. Disagreements during this process were discussed until a consensus was achieved. Disconfirming evidence was actively sought throughout. All interviewees were sent a copy of the preliminary analysis for comment. Emerging ideas and themes were modified in response. ${ }^{13} \mathrm{~A}$ cross-comparative case-study report based on the contents of the individual case-study reports was produced at each time point. The final analysis represented a synthesis of the findings from the four cross-comparative case-study reports, service user, carer, trust-level reports, site-specific documentation and field notes that described service contexts.

\section{Results}

All 14 early intervention services in the West Midlands (population 6 million) participated in the study. Each was at a different stage of development. Two services consisted of a single team manager in place to set the service up and did not actively recruit patients throughout the study period. One team had been established for 10 years and another for 3 years. All other services had been in existence for 6-18 months. The longest established service had the greatest number of whole-time-equivalent staff $(n=25)$. Team size varied but each keyworker was expected to have an active case-load of 15 service users. Ten services adopted a stand-alone team model where team members worked solely with individuals with first-episode psychosis. Two of the twelve active services, both in rural areas and where funding was uncertain, adopted a 'hub and spoke' or 'hybrid' approach. In the hub and spoke model, early intervention workers were sited within a community mental health team; in the 'hybrid' model, the team incorporated elements of both stand-alone and hub and spoke models.

All early intervention services focused on biological issues such as the need to treat presenting psychotic symptoms with low-dose antipsychotic medication; psychological perspectives, including providing individuals with the skills they needed to help them understand their psychosis; and social aspects such as help accessing vocational and social activities. All services also offered family psychoeducation.

A total of 162 interviews took place over the 18 months. Where possible, the same three to five individuals at each site were interviewed at each time point. The data, therefore, represent the longitudinal views of over 50 early intervention service team managers and staff. About $50 \%$ of the interviewees were community psychiatric nurses and $25 \%$ were psychologists or psychiatrists. Other interviewees were from social work, youth work, occupational therapy or community development work backgrounds.

Of the 12 active teams, 9 completed the fidelity scale. Scores ranged from 169 to 231 (mean score of 200 from a possible 256), suggesting good fidelity with policy guidance. The lowest score was for the service adopting a hybrid model.

Of the 62 strategic health authority mental health leads, primary care trust mental health commissioners and mental health trust and social care trust chief executives, 42 (68\%) agreed to be interviewed.

Overall, 479 service users were incepted into the 12 active early intervention services over a 2-year period (17-98 per service); 339 (70.7\%) were male and 269 (55.9\%) were White. A total of 32 service users, purposively sampled on age, gender and ethnicity from 11 of the 12 active services, and 18 carers were interviewed before data saturation was complete. Service users who were interviewed were aged between 15 and 34 (mean age 23 years for both genders), 22 were White British and 12 were female. All carers were parents aged between 35 and 72 years.

Of service users, $90.6 \%$ remained engaged at 12 months after inception into the early intervention service: $12(2.5 \%)$ had been discharged, $19(4.0 \%)$ had disengaged and $14(2.9 \%)$ had never engaged fully with the service.

\section{New approaches to care}

Engagement was seen as the hallmark of an early intervention service. The foundations were good interpersonal as well as professional skills and focusing on issues relevant to a young clientele. Education and employment issues were priorities for many service users and therefore teams often addressed these before conversations about medication. Accessibility was also a vital element. Many team members used text messages to communicate with services users and deliberately met in low-stigma settings such as the local café.

Eleven early intervention services were multidisciplinary. Most staff shared jobs and responsibilities regardless of professional background. This once again reflected the strong emphasis given to engagement by all teams, which meant that apparently 'mundane' tasks such as a visit to the cinema as part of the keyworker role, lifts to the dentist or to the pharmacist were seen as important in keeping service users in contact with the service and helping them cope with everyday things at a point in their life when these could feel impossible to achieve. Psychologists and psychiatrists were, however, the least likely to work in this way since they were perceived as offering profession-specific interventions and were relatively few in number.

Most services actively tried to distance themselves from being seen by users as part of the wider psychiatric system. However, this 'part of yet different from' approach led to tensions within some areas with generic community mental health teams. A minority of services, all newly emerging services, felt that some community mental health teams perceived them as elitist, as poaching staff and as having a less intensive workload because of their smaller case-worker:client ratios. Most saw this as a natural response to 
change, and appreciated that perhaps some community mental health teams had previously enjoyed working with young people with a first episode of psychosis. Better communication strategies between mental health teams were described as key in helping different teams appreciate their relative strengths. This was also seen as critical in terms of developing exit strategies at the end of the 3 years with the early intervention service back into an inevitably less personal community mental health team service.

All services felt that developmental work was a core part of their team identity and helped to further differentiate them from other mental health services. Two of the more securely financed services ran regular community psychosis detection training and anti-stigma campaigns. Most of the other services focused on developmental work in primary care and schools, where they felt they could make most impact on duration of untreated psychosis within their limited development budget (online supplement 1).

\section{Importance of context}

A number of demographic factors, particularly relative deprivation and geography, were noted as influencing the implementation of early intervention services. Teams operating in relatively deprived areas were more likely to highlight challenges presented by service users' multiple complex needs, including poor housing and high local unemployment rates. This created additional work in liaising with a range of services, so that poverty, in a sense, created additional barriers to recovery. Teams in deprived areas were also more likely to report working with 'hard-to-reach' groups such as asylum seekers, refugees and migrant communities (online supplement 2). This again led to additional work, not only in terms of accessing good-quality translators who understood mental health issues and could ensure subtleties were not lost in translation, but also in creating mutual cultural understanding.

The influence of geography was felt more keenly in rural services, where balancing travel time and face-to-face work with service users was a constant issue. Access to support services was also more limited than in urban areas. Indeed there were far more descriptions of partnership working with the voluntary and community sector within urban teams. A more unexpected finding was the problems created for confidentiality within small and often close-knit rural communities (online supplement 2).

\section{The influence of commissioners}

Most commissioners, usually senior managers within the organisation that held the funding for developing services, felt they were relatively inexperienced in commissioning early intervention services. Although partnership working across health and social care was seen as vital in developing services, many primary care trust commissioners cited difficulties in making this happen because of poor relationships between different agencies, insufficient resources and recurring organisational restructuring. The least experienced commissioners described particular problems engaging with child and adolescent mental health services, and voluntary and community sector organisations (online supplement 3).

A significant minority of the primary care trust commissioners described problems in commissioning because they felt the primary care trust placed a low priority on mental health. They described an unexpected feeling of stigma attached to their own role which they felt reduced their potential to develop intra- and inter-organisational relationships and ring-fence budgets for mental health services (online supplement 3 ).

\section{Strategies for survival in uncertain times}

The major barrier to developing services from an early intervention service perspective was uncertainty over funding. Commissioner inexperience was frequently commented on by team managers, who felt they had little understanding of the ethos, development and delivery of services, leading to delayed decision-making and problems in securing ongoing funding. Most team managers and particularly those in newly emerging teams, felt commissioners were too focused on ensuring that case-loads of 15 patients per keyworker were met, a target that was tied in part to securing the following year's funding. Early intervention services responded to the tensions between quality and quantity and uncertainty over funding streams in a number of ways. In the first round of interviews, many team managers described how services relied on staff good will to continue working in such an intensive manner. Team members, however, described the negative effect on morale of working at or beyond capacity for long periods of time to meet targets and ensure further resources were made available. There were also worries about retention of staff and burnout (online supplement 4).

At the second interviews, most team managers talked about a need to reconfigure as a hub and spoke model to save money while attempting to maintain fidelity to the early intervention service framework. During the next 6 months, however, most services managed their workload through tightening age eligibility criteria (one service), discharging service users earlier than the intended 3-year period (three services) or imposing waiting lists (three long-established services). They were aware that these adaptations rendered them non-compliant with Department of Health policy implementation guidance and, in the case of invoking waiting lists, was recognised as inimical to early intervention. All but two teams also spent less time on developmental work in the community than they would have liked. During the final round of data collection, no service operated a waiting list and most were trying to find different ways to continue by, for example, working in partnership with other organisations and finding alternative sources of additional funding (online supplement 4).

\section{Service user and carer views}

Almost all service users were anxious at the prospect of referral to a mental health service but described the reality of engaging with early intervention services as positive. Fourteen of the service users who were interviewed were initially referred after an in-patient admission on a psychiatric ward. These service users compared their experiences of hospital-based care and early intervention services, and described the former as places where they were treated like children, not listened to, were unable to influence decisions and where staff constantly changed. Early intervention services, in contrast, were staffed by people who were 'consistent', 'knew what they were doing', 'cared', 'weren't pushy but listened', and were 'very helpful and gave lots of information' (online supplement 5).

Many noted that they themselves had held negative views of people with mental illness before their own episode of psychosis and described being ashamed and embarrassed initially about their own diagnosis. The early intervention services had helped by giving them information that addressed their stereotypes, reduced their fears about the future and increased their selfconfidence so they were more able to talk to friends and employers about their illness.

Most carers were also happy with the care provided by the early intervention services, often comparing them positively with services they had previously been in contact with. They highlighted the availability of team members out of hours and the hope for recovery they were given. Many mourned their 
own loss of independence since the diagnosis and valued the extra time and support offered to them throughout the illness. Some felt the timely and high-quality interventions had saved lives (online supplement 5).

\section{Discussion}

This in-depth longitudinal study tracked changes in early intervention services over time to gain multiple views of commissioning, delivering and experiencing such services. It provides a series of complex contextualised unfolding stories that may hold lessons for the implementation and development of other health services nationally and internationally. It shows that the number of services has increased in response to policy directives; that they include the key structural elements required by national early intervention service implementation guidance; can effectively engage service users over a 12-month period; and are highly valued by service users and carers. However, there are tensions between providing a quality service and meeting case-load targets linked to future funding and gaps between stated policy and actual practice.

Early intervention services managed these through a variety of survival strategies. The situation appeared to be exacerbated by a lack of experience and understanding of the need for consistent funding at commissioner level.

We had expected more negative comments from users and carers about the high and sustained intensity of the services and the possible sense of intrusion from the community, predominantly home-based, intervention. None had yet experienced the step down in care around discharge, which may conceivably be experienced as a relief and a threat. This is an important future question and challenge for the policy guidance.

\section{Strengths and weaknesses in relation to other studies}

Two trials have demonstrated that integrated intensive services at an early stage of psychosis can lead to improved clinical outcomes, including fewer psychotic symptoms ${ }^{14}$ and lower relapse rates. ${ }^{15}$ The findings add to these and other previous studies on early intervention services, by helping to explain the elements and methods that underpin their success, including the importance of developing a youth-friendly service with a recovery focus. ${ }^{16}$ Engagement with and advocacy for service users have also been described as critical issues in developing early intervention services. ${ }^{17} \mathrm{~A}$ lack of understanding and experience at commissioner level are recognised barriers to good commissioning in a range of healthcare contexts. ${ }^{18}$

Few previous studies of early intervention services have reported such high service engagement at 12 months. Indeed the UK trial of early intervention reported contact rates at 18 months with community mental health teams of $59 \% .{ }^{15}$ The positive views of service users and carers provide a contrast with the negative views in a national survey of users with first-episode psychosis and their carers in 2002 and more recent Scottish data on the needs of and service provision for young people with adolescent-onset first-episode psychosis receiving care from mainstream mental health services. ${ }^{19}$

\section{Limitations}

Study limitations include the method of approaching service users and carers. Although the research team purposively selected service users to be invited to take part in an interview, the keyworkers in each team then approached them to gain initial consent. This approach might potentially have excluded the recruitment of users with more critical opinions. We also only had ethical approval to interview carers if services users gave their consent, which, once again, may have excluded carers with the most strongly held or critical opinions. Service engagement was assessed through looking at care records at 12 months to see how many service users were still in active weekly contact with their keyworker, rather than through a service-user-completed engagement scale. We did not collect data on the views of community mental health team members on early intervention services which, with hindsight, could have provided interesting perspectives on perceptions of elitism noted by some of the interviewees. Qualitative methods enabled a more in-depth understanding of the challenges faced by different teams. The generalisability of the project findings derives not from the representativeness of the sample but from the concepts that are relevant to other settings and patient groups. ${ }^{20}$

\section{Implications for clinicians and policy-makers}

Early intervention services now have a stronger evidence base in terms of effectiveness than all other recent service-level changes in mental healthcare, including assertive community treatment ${ }^{21}$ and home treatment services. ${ }^{22}$ There is, however, still scepticism about service implementation from parts of the mental health system. ${ }^{23}$ This in-depth study may help to allay some of this criticism by demonstrating the evolutionary and generalisable mechanisms, rooted in good interpersonal relationships, commitment and flexibility in adversity, that underpin the success of early intervention.

In healthcare systems where patient choice and satisfaction are increasingly recognised as important, ${ }^{24}$ the positive views of early intervention services from service users and carers, particularly in comparison with views of more traditional generic or hospitalbased services, are encouraging for policy-makers, funders and clinicians. This study of routine service provision, outside of the necessarily controlled setting of a trial, has also demonstrated that early intervention services are able to engage young people with first-episode psychosis very effectively. Other mental health teams may find the engagement strategies and new approaches to care helpful in informing their own service delivery plans.

However, the tensions created for staff in having to meet performance targets related to case-loads, as well as pursuing developmental work in the community and supporting often distressed family members, led to the development of a series of pragmatic survival strategies without knowing the consequences for user outcomes. Although most teams found a middle ground over time, there were inevitable consequences for team morale and patient care, including the oxymoron of a waiting list for an early intervention service, as the teams struggled to balance differing pressures and demands. Early intervention services are not unique in experiencing such tensions and there are generic implications for service planners about the unintended consequences of performance targets in a healthcare setting. Finally, consistent funding that recognises the importance of developmental as well as case-load work is required to ensure the continued implementation of services across England.

Helen Lester, MBBCh, National Primary Care Research \& Development Centre, Manchester; Max Birchwood, DSc, School of Psychology, College of Life and Environmental Science; Stirling Bryan, PhD, Health Services Management Centre, Environmental Science; Stirling Bryan, PhD, Health Services Management Centre,
University of Birmingham; Elizabeth England, MBBS, Helen Rogers, PhD, Namita Sirvastava, MSc, Department of Primary Care, College of Medical and Dental Sciences, University of Birmingham, UK

Correspondence: Professor Helen Lester, National Primary Care Research \& Development Centre, 5th Floor Williamson Building, Oxford Road, Manchester M13 9PL, UK. Email: Helen.lester@manchester.ac.uk

First received 9 Apr 2008, final revision 26 Aug 2008, accepted 22 Oct 2008 


\section{Funding}

This study was funded by the National Institute for Health Research Service Delivery and Organisation Programme. H.L. is the guarantor of the paper. M.B. was joint grant holder S.B. was a grant holder. H.L. is independent from the funding body and the views expressed in this paper have not been influenced by the funding source.

\section{Acknowledgements}

We thank Mrs Nicola Jones-Morris, Ms Julie Richards and Dr Effy Tzemou who each helped to collect some of the data from early intervention services across the West Midlands.

\section{References}

1 Perälä J, Suvisaari J, Saarni SI, Kuoppasalmi K, Isometsä E, Pirkola S, et al. Lifetime prevalence of psychotic and bipolar I disorders in a general population. Arch Gen Psych 2007; 64: 19-28.

2 Rethink. Reaching People Early. Rethink, 2002.

3 Yung AR, Organ BA, Harris MG. Management of early psychosis in a generic adult mental health service. Aust N Z J Psychiatry 2003; 37: 429-36.

4 McGlashan TH. Duration of untreated psychosis in first episode schizophrenia: marker or determinant of course? Biol Psych 1999; 46: 899-907.

5 Norman RMG, Malla AK. Duration of untreated psychosis: a critical examination of the concept and its importance. Psychol Med 2001; 31 381-400.

6 Harrison G, Hopper K, Craig T, Laska E, Siegel C, Wanderling J, et al. Recovery from psychotic illness: a 15- and 25-year international follow-up study. Br J Psychiatry 2001; 178: 506-17.

7 Department of Health. The National Plan for the NHS. Department of Health 2001.

8 Keen J, Packwood T. Using case studies in health services policy and research In Qualitative Research in Health Care (eds C Pope, N Mays). BMJ Publishing, 2000.

9 Yin R. Case Study Research: Design and Methods (2nd edn). Sage, 1994
10 Department of Health. Early intervention in psychosis. In The Mental Health Policy Implementation Guide: 43-61. Department of Health, 2001.

11 Glaser BG. Theoretical Sensitivity: Advances in the Methodology of Grounded Theory. Sociology Press, 1978.

12 Silverman D. Interpreting Qualitative Data. Methods for Analysing Talk, Text and Interaction. Sage, 1997.

13 Petersen L, Jeppesen $\mathrm{P}$, Thorup A, Abel MB, Øhlenschlaeger J, Christensen $\mathrm{T \varnothing}$, et al. A randomised multicentre trial of integrated versus standard treatment for patients with a first episode of psychotic illness. BMJ 2005; 331: 602

14 Craig TK, Garety P, Power P, Rahaman N, Colbert S, Fornells-Ambrojo M, et al. The Lambeth Early Onset (LEO) Team: randomised controlled trial of the effectiveness of specialised care for early psychosis. BMJ 2004; 329 : 1067-71.

15 Edwards J, McGorry P, Pennel K. Models of early intervention in psychosis: analysis of service approaches. In Early Intervention and Psychosis (eds M Birchwood, D Fowler, C Jackson). Wiley, 2000.

16 Edwards J, Harris MG, Bapat S. Developing services for first-episode psychosis and the critical period. Br J Psychiatry 2005; 187: s91-7.

17 Peck E, Greatley A. Mental Health Priorities for Primary Care. King's Fund Centre for Mental Health Services Development, 1999.

18 Boeing L, Murray V, Pelosi A, McCabe R, Blackwood D, Wrate R. Adolescentonset psychosis: prevalence, needs and service provision. $\mathrm{Br} J$ Psychiatry 2007; 190: 18-26.

19 Green J. Generalisability and validity in qualitative research. BMJ 1999; 319: 421.

20 Marshall $\mathrm{M}$, Creed $\mathrm{F}$. Assertive community treatment: is it the future of community care in the UK? Int Rev Psychiatry 2000; 12: 191-6.

21 Burns T, Knapp M, Catty J, Healey A, Henderson J, Watt H, et al. Home treatment for mental health problems: a systematic review. Health Technol Assess 2001; 5: 1-139.

22 Pelosi AJ, Birchwood M. Is early intervention for psychosis a waste of valuable resources? Br J Psychiatry 2003; 182: 196-8.

23 Healthcare Commission. Survey of Users of Mental Health Services 2007. Healthcare Commission, 2007. 